\title{
Chinese Herbal Formula Huo-Luo-Xiao-Ling Dan Protects against Bone Damage in Adjuvant Arthritis by Modulating the Mediators of Bone Remodeling
}

\author{
Siddaraju M. Nanjundaiah,, David Y.-W. Lee, ${ }^{2}$ Brian M. Berman, ${ }^{3}$ and Kamal D. Moudgil ${ }^{1}$ \\ ${ }^{1}$ Department of Microbiology and Immunology, University of Maryland School of Medicine, HSF-1, Suite 380, \\ 685 West Baltimore Street, Baltimore, MD 21201, USA \\ ${ }^{2}$ Mailman Research Center, McLean Hospital, Harvard Medical School, Belmont, MA 02478, USA \\ ${ }^{3}$ Center for Integrative Medicine, University of Maryland School of Medicine, East Hall, 520 West Lombard Street, \\ Baltimore, MD 21201, USA
}

Correspondence should be addressed to Kamal D. Moudgil; kmoud001@umaryland.edu

Received 20 December 2012; Revised 8 March 2013; Accepted 8 March 2013

Academic Editor: Vincenzo De Feo

Copyright (C) 2013 Siddaraju M. Nanjundaiah et al. This is an open access article distributed under the Creative Commons Attribution License, which permits unrestricted use, distribution, and reproduction in any medium, provided the original work is properly cited.

\begin{abstract}
Huo-luo-xiao-ling dan (HLXL) is an herbal mixture that has long been used in traditional Chinese medicine for the treatment of rheumatoid arthritis (RA) and other inflammatory disorders. Despite the availability of potent conventionally used drugs for RA, their limited efficacy in a proportion of patients coupled with their high cost and severe adverse effects has necessitated the search for novel therapeutics for this debilitating disease. Further, the control of both inflammation and bone damage is essential for effective management of arthritis. The aim of our study was to evaluate the efficacy of HLXL against arthritic bone damage in adjuvant arthritis (AA) model of RA. Our results show that HLXL treatment suppressed inflammatory arthritis and reduced bone and cartilage damage in the joints of arthritic Lewis rats. HLXL-induced protection against bone damage was mediated primarily via inhibition of mediators of osteoclastic bone remodeling (e.g., receptor activator of nuclear factor kappa-B ligand; RANKL), skewing of RANKL/osteoprotegerin (OPG) ratio in favor of antiosteoclastic activity, reduction in the number of osteoclasts in the arthrodial joint's bone, and inhibition of cytokine production and MMP activity. Our results suggest that HLXL might offer a promising alternative/adjunct treatment for both inflammation and bone damage in RA.
\end{abstract}

\section{Introduction}

Rheumatoid arthritis (RA) is a global autoimmune disease, affecting about 1 percent of the population in USA and Europe, for example [1]. The disease is characterized by chronic inflammation of the synovial tissue in the joints $[1,2]$. Uncontrolled disease leads to significant disability and deformities of the hands and feet. A variety of potent antiarthritic drugs, including biologics, have been used for the treatment of RA over the past decade or so $[1,3]$. However, these conventionally used drugs have limitations. Their efficacy may be limited to a subset of patients, and their use may be associated with severe adverse reactions $[3,4]$. In addition, these drugs are rather expensive. Accordingly, there is a continued search for newer therapeutic agents for RA. Natural plant products belonging to the traditional Chinese medicine (TCM) represent a diverse collection of potential therapeutic agents for a wide variety of diseases including RA [5-7]. TCM represents one of the components of complementary and alternative medicine (CAM). The popularity of CAM is gradually increasing in USA and other industrialized countries. For example, according to one survey, approximately 38 percent of adults and 12 percent of children used CAM remedies for different health needs annually [8]. Thus, there is a need to optimize the composition of a herbal CAM and to define its mechanism of action before it can be considered for further trials for the treatment of RA in the near future. The present study is aimed at fulfilling these important objectives for a TCM herbal mixture for the treatment of arthritis. 
Huo-luo-xiao-ling dan (HLXL), a traditional Chinese medicine (TCM) herbal formula and its modified versions have long been used in folk medicine to treat inflammatory arthritis or joint pain, referred to as the "Bi syndrome" $[9,10]$. The use of a combination of multiple herbs is designed to exploit the additive or synergistic activities of individual herbs, as well as to balance or neutralize the toxic effects of certain herbal components by others in the mixture [5]. In our previous studies using the rat adjuvant arthritis (AA) model of human RA [11-13], we have tested the anti-inflammatory properties of a modified version of the originally used HLXL. The treatment of Lewis rats with the modified HLXL preparation was effective in reducing inflammatory arthritis [11-13]. However, it remained to be determined whether HLXL had any effect on bone and cartilage damage associated with inflammatory arthritis. The rationale for this testing lies in the fact that certain conventionally used antiarthritic agents may efficiently suppress inflammation but not bone damage $[14,15]$; the opposite may be the case for other antiarthritic agents [16-19]. Therefore, for an ideal antiarthritic agent, it is imperative that its effects are tested not only on inflammation but also on bone damage. Accordingly, the mechanism underlying the influence of HLXL on bone remodeling needed to be unraveled. The present study was aimed at filling these vital gaps.

We describe in this study the results of testing HLXL in arthritic Lewis rats. We examined and compared the arthritic paws of HLXL-treated versus vehicle-treated Lewis rats by arthritic scores, radiography, and histomorphometry. We also tested the synovial-infiltrating cells (SIC) for the production of mediators of bone remodeling such as receptor activator of nuclear factor $\mathrm{kB}$ ligand (RANKL), osteoprotegerin (OPG), granulocyte-macrophage colony stimulating factor (GM-CSF), osteopontin (OPN), and insulin-like growth factor (IGF). Of these, RANKL promotes osteoclastogenesis, whereas OPG serves as a decoy receptor for RANKL and thereby antagonizes the effect of RANKL. Bone damagerelated cytokines (IL-1 $\beta$ and IL-18) and matrix metalloproteinases (MMPs) were also tested. Our results show that HLXL affords protection against bone and cartilage damage in the joints of arthritic rats via modulating the mediators of bone remodeling. Thus, this herbal TCM targets both inflammation and bone damage in arthritis.

\section{Materials and Methods}

2.1. Animals. Five- to six-week-old male Lewis (LEW/Hsd) $\left(\right.$ RT. $\left.1^{1}\right)$ rats were used in this study. Rats were purchased from Harlan Sprague-Dawley (HSD) (Indianapolis, IN, USA) and then maintained in the animal care facility of the University of Maryland School of Medicine, Baltimore, MD, USA. All experimental procedures performed on these rats were in accordance with the guidelines of the Institutional Animal Care and Use Committee (IACUC).

2.2. Composition and Characteristics of HLXL. The herbal formula huo-luo-xiao-ling (HLXL) dan tested in this study is similar to that used in our previous studies [20-23], and it consists of a mixture of 11 well-defined herbs, namely, Ruxiang (Boswellia carterii Birdw.), Qianghuo (Notopterygium incisum Ting ex H.T. Chang), Danggui (Angelica sinensis (Oliv.) Diels), Chishao (Paeonia lactiflora Pall.), Gancao (Glycyrrhiza uralensis Fisch.), Yanhusuo (Corydalis yanhusuo W.T. Wang.), Danshen (Salvia miltiorrhiza Bge.), Chuanxiong (Ligusticum chuanxiong S.H. Qiu.), Qinjiao (Gentiana macrophylla Pall.), Guizhi (Cinnamomum cassia Presl.), and Duhuo (Angelica pubescens Maxim). We have previously reported in detail the methods for the preparation of HLXL, for the characteristics of its component herbs and for the assessment of its toxicity [21, 22]. The batch of HLXL used in this study was thoroughly characterized by HPLC fingerprinting as in our earlier studies [11-13, 20-23]. The HPLC profile included the peak shapes, numbers, intensities, and retention times of all individual compounds (data not shown). In addition, two marker compounds, Swertiamarin and paeoniflorin, served as references for quality control purposes.

2.3. Treatment of Arthritic Rats with HLXL. Lewis rats were immunized subcutaneously (s.c.) at the base of the tail with $1 \mathrm{mg} /$ rat heat-killed M. tuberculosis H37Ra (Mtb) (Difco, Detroit, MI, USA) in $200 \mu \mathrm{L}$ of mineral oil (Sigma-Aldrich). Following the onset of arthritis, these rats were randomly divided into two groups (experimental and control). Finely powdered HLXL was suspended in water, and it was fed $(2.3 \mathrm{~g} / \mathrm{kg})$ to the experimental group of rats using a gavage needle (FNC-16-3, Kant Scientific Corporation, Torrington, CT, USA) beginning on the day of onset of arthritis (d 10) and then continued up to the peak phase of AA (d 18). On the corresponding days, the control group of rats received Water (the vehicle) by gavage. All rats were examined and graded regularly for the severity of arthritis as described earlier $[24,25]$. The test samples were collected from rats when the disease reached the peak phase ( $\mathrm{d} 18)$ in controls.

2.4. Histological Examination of Hind Paws of Rats. The hind paws were harvested from Lewis rats on d 18 after Mtb immunization and immersed for $9 \mathrm{~d}$ in Cal-Ex Decalcifying solution CS510-1D (Fisher Scientific, Fair Lawn, NJ, USA). Thereafter, the paws were immersed in $70 \%$ ethanol for 5 $\mathrm{d}$ and then embedded in paraffin, sectioned serially using a microtome, and mounted on microscope slides. Then the sections were stained either with hematoxylin and eosin (H\&E) (Histology Core, UMB) [26] or with safranin O [27, 28]. Histopathological changes in the joints like synovial hyperplasia, pannus formation, and bone damage were observed under a microscope (Nikon Eclipse E800 Microscope, Nikon Industries Inc. Melville, NY, USA) using the Spot Imaging Software (Diagnostic Instruments Inc., Sterling Heights, MI, USA) and digital images were obtained.

\subsection{Tartrate-Resistant Acid Phosphatase (TRAP) Staining.} The unstained, mounted microtome sections (as described above) were dehydrated in graded concentrations of ethanol and xylene and fixed for 2 min using 3.7\% formaldehyde. The sections were washed with deionized water and were incubated in the reaction mixture (acid phosphatase, Leukocyte 
(TRAP) Kit, Sigma-Aldrich) at $37^{\circ} \mathrm{C}$ in a humid and lightprotected incubator for $1 \mathrm{~h}$ as directed by the manufacturer. Thereafter, the sections were washed again 3 times with distilled water. Later, the sections were counter-stained with hematoxylin and observed under a microscope using the Spot Imaging Software, and digital images were obtained.

2.6. Bone Histomorphometry of Hind Paws. TRAP-stained hind paw sections of rats ( $n=5$ per group) were used to perform bone histomorphometry with the Osteomeasure Bone Histomorphometry system (Osteometrics, Atlanta, GA, USA) linked to a Nikon Eclipse 50i inverted microscope and a Sony CCD video camera [29]. The analyses were performed on serial transverse sections through the talus $(n=6)$. Bone volume versus total tissue volume (BV/TV), the number of osteoclasts per tissue area (N.Oc/T.Ar), active resorption per bone surface area based on the ratio of osteoclast surface/bone surface area (Oc.S/BS), and the number of osteoclasts per bone perimeter (N.Oc/B.Pm) were assessed. Histomorphometric parameters follow the recommended nomenclature of the American Society for Bone and Mineral research [30].

2.7. Radiographic Assessment of Arthritis in Hind Paws of Rats. The severity of AA was assessed blindly on d 18 by radiography. High-resolution digital radiography $(40 \mathrm{kV}$, $12 \mathrm{~s}$ ) of hind limbs was performed on rats under ketaminexylazine anesthesia using a Faxitron Digital X-ray system (Faxitron X-Ray, Lincolnshire, IL, USA) [29].

2.8. Preparation of Synovial-Infiltrating Cells (SIC), Their Restimulation with Mtb, and Testing for Mediators of Bone Damage. SIC (total SIC) were collected by cutting open the hind paw (ankle) joints of Mtb-immunized rats on d 18 using a sterile surgical blade. These SIC were washed 3-4 times with HBSS and then were cultured in a 12-well plate using DMEM supplemented with $5 \%$ fetal bovine serum (FBS), $2 \mathrm{mML}$ glutamine, $100 \mathrm{U} / \mathrm{mL}$ penicillin $\mathrm{G}$ sodium, and $100 \mu \mathrm{g} / \mathrm{mL}$ streptomycin sulfate. The nonadherent cells were removed after 90 min by washing the culture dish with HBSS [25]. The remaining cells (adherent SIC) were restimulated for $24 \mathrm{~h}$ with $\mathrm{Mtb}$ sonicate $(10 \mu \mathrm{g} / \mathrm{mL})$ in DMEM containing $5 \%$ FBS. Thereafter, culture supernatant was collected and tested for mediators of bone remodeling by Multiplex assay in the Cytokine Core Facility (University of Maryland, Baltimore, MD, USA) using the Luminex 100 analyzer (Luminex Corp., Austin, TX, USA). In addition, the culture supernatant was tested for matrix metalloproteinases (MMPs) as described elsewhere [27].

2.9. Statistical Analysis. The data were expressed as mean \pm SEM. Student's $t$-test and ANOVA Bonferroni's post hoc method were used to assess the significance of differences using GraphPad Prism version 4.0. A $P$ value of $<0.05$ was considered statistically significant.

\section{Results}

3.1. HLXL Suppresses Inflammation and Tissue Damage in the Joints of Arthritic Rats. Arthritic Lewis rats were fed daily with HLXL (in water, by gavage) beginning at the onset (d 10) of AA and then continued up to the peak phase ( $d$ 18) of the disease, whereas the corresponding control rats received water by gavage. There was a significant reduction in the severity of clinical arthritis (see Figure S1(a) in Supplementary Material available online at http://dx.doi.org/10.1155/2013/429606). Histological examination revealed significant reduction of pannus formation, synovial mononuclear cell infiltration, and bone destruction in HLXL-treated rats compared to control rats (Supplementary Figures 1(b)-1(d)). In parallel, histological sections subjected to TRAP staining (for osteoclasts) (Figures 1(e) and $1(\mathrm{f})$ ) and safranin-O staining (for cartilage) (Figures $1(\mathrm{~g})$ and $1(\mathrm{~h})$ ) showed reduction in osteoclasts as well as cartilage damage. On $\mathrm{d} 18$, the hind paws of rats were subjected to radiological examination; the radiographs showed reduction in the inflamed soft tissue around the joints and bone damage in HLXL-treated rats compared to control rats (Figures 1(a)$1(d))$.

3.2. HLXL Suppresses Bone Loss and Osteoclast Number in Arthritic Lewis Rats. AA is characterized by bone resorption, which is evident from histomorphometric examination of hind paw sections of arthritic rats compared with those of naïve rats (Supplementary Figure 2). Therefore, we tested whether HLXL treatment altered these histomorphometric parameters. For this, subchondral bone loss and osteoclast numbers in the talus of the hind paw joints of the experimental and control rats were analyzed. We observed a significant reduction in the subchondral bone loss and increased bone volume in HLXL-treated rats compared to the control rats (Figure 2(a)). Histomorphometric analysis of tartrateresistant-acid-phosphatase- (TRAP-) stained joint sections revealed that the number of osteoclasts (Figure 2(b)) and the corresponding active resorption surfaces (Figure 2(c)) were reduced in HLXL-treated rats compared to those of control rats. Osteoclast number/bone perimeter was also reduced after treatment with HLXL when compared to controls (Figure 2(d)).

3.3. HLXL Regulates Mediators of Bone Remodeling in Arthritic Rats. To determine the mechanisms underlying the observed effects of HLXL on bone remodeling, we tested the effect of HLXL on the mediators of bone remodeling (RANKL, OPG, GM-CSF, OPN, and IGF) in experimental and control rats. These mediators were measured in culture supernatants of synovium-infiltrating cells (SIC), which had been restimulated with sonicated, heat-killed $M$. tuberculosis H37Ra (Mtb sonicate) (Figure 3). There was a significant decrease in all of the above-mentioned bone remodeling mediators tested in SIC of HLXL-treated rats compared to those of control rats (Figure 3). Though both RANKL and OPG levels were reduced but to different extents, leading to deviation of the RANKL/OPG ratio in favor of antiosteoclastic activity in HLXL-treated rats compared to control rats. 


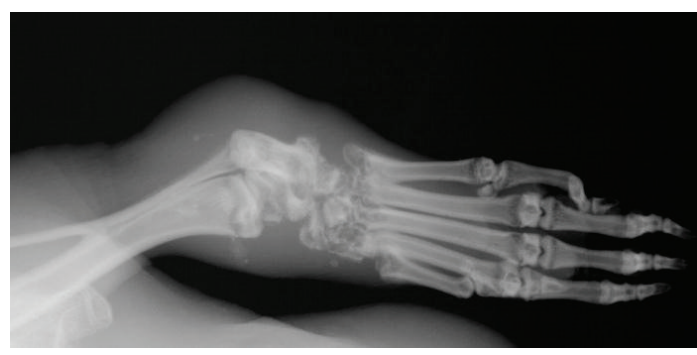

(a)

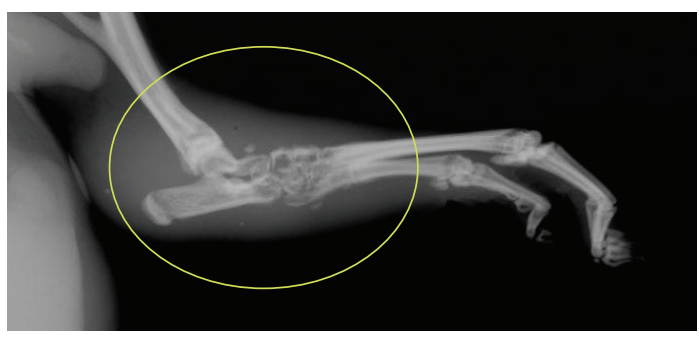

(c)

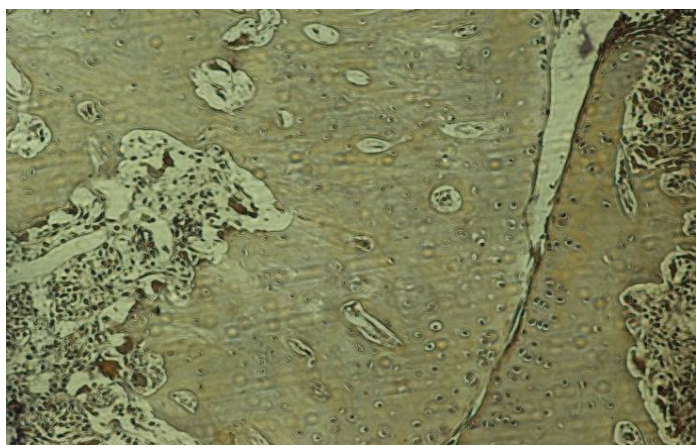

(e)

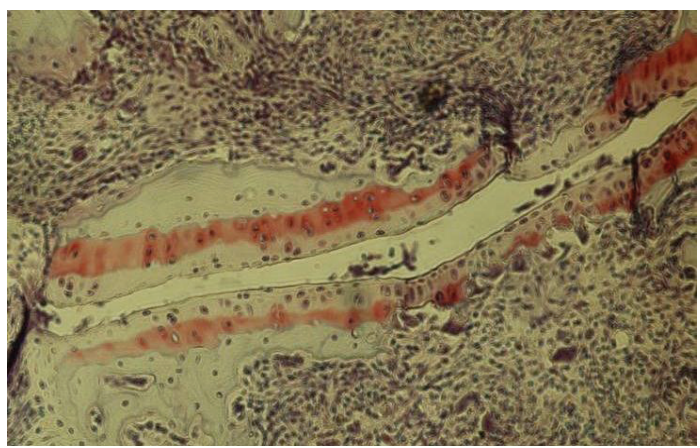

(g)

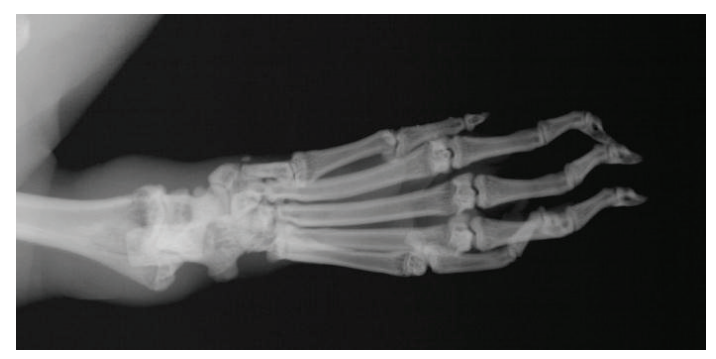

(b)

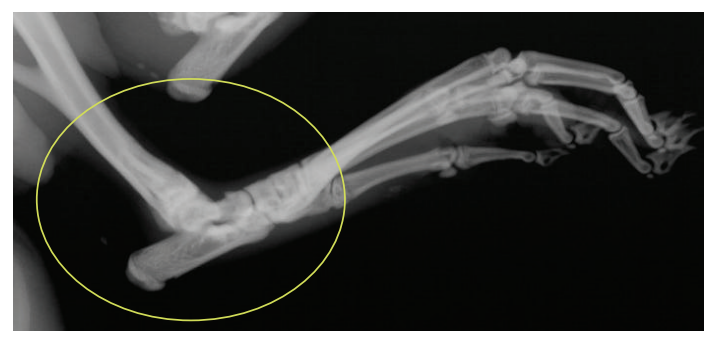

(d)

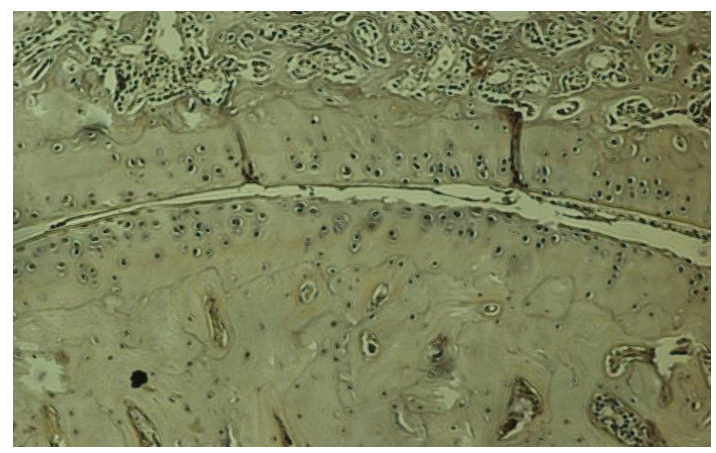

(f)

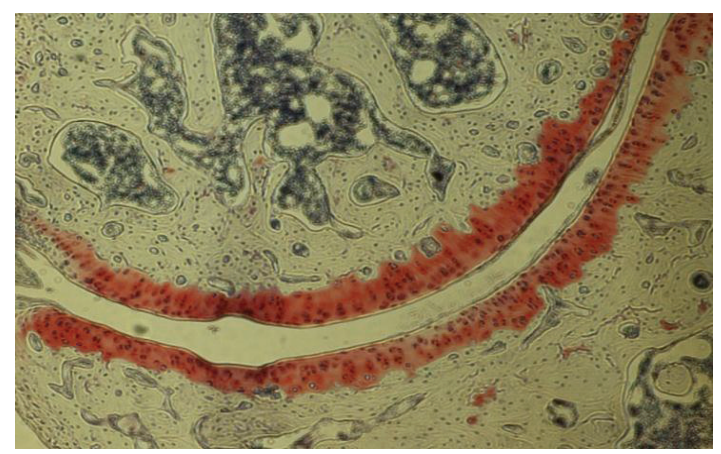

(h)

FIGURE 1: HLXL attenuates arthritic inflammation and joint damage in Lewis rats. A group each ( $n=4$ per group) of Mtb-immunized Lewis rats was fed either Water (Vehicle; (a), (c), (e), (g)) or HLXL (2.3 g/kg; (b), (d), (f), (h)), beginning at the onset of AA and then continued through d 18 after Mtb injection. (a), (b) (frontal view) and (c), (d) (lateral view) depict representative radiographs of hind limbs on d 18. Representative micrographs of tartrate-resistant-acid-phosphatase- (TRAP-) stained ((e), (f)) and safranin O-stained ((g), (h)) sections of the hind paw joints of control and HLXL-treated rats are shown.

3.4. HLXL Treatment Inhibits Antigen-Induced Proinflammatory Cytokine Response and MMP Activity in Arthritic Rats. IL-18 and IL- $1 \beta$ are proinflammatory cytokines that have a significant effect on bone remodeling. Therefore, we tested the levels of these two cytokines (as proteins) in SIC that were harvested from HLXL-treated and control arthritic rats and then restimulated in vitro for $24 \mathrm{~h}$ with sonicated Mtb. There was a significant decrease $(P<0.05)$ in the level of IL-18 as well as IL- $1 \beta$ in HLXL-treated rats compared with Water-treated rats (Figure 4, left panel). We also tested in SIC the levels of MMPs (MMP-2 and MMP-9), one of the key mediators of tissue damage in arthritis (Figure 4, right panel). 


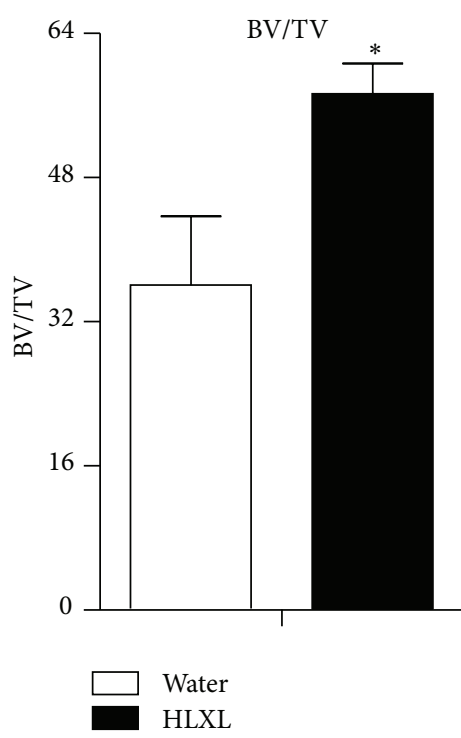

(a)

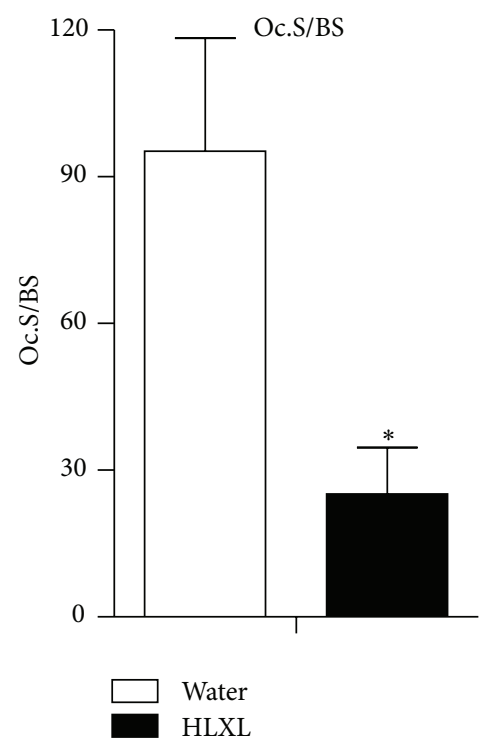

(c)

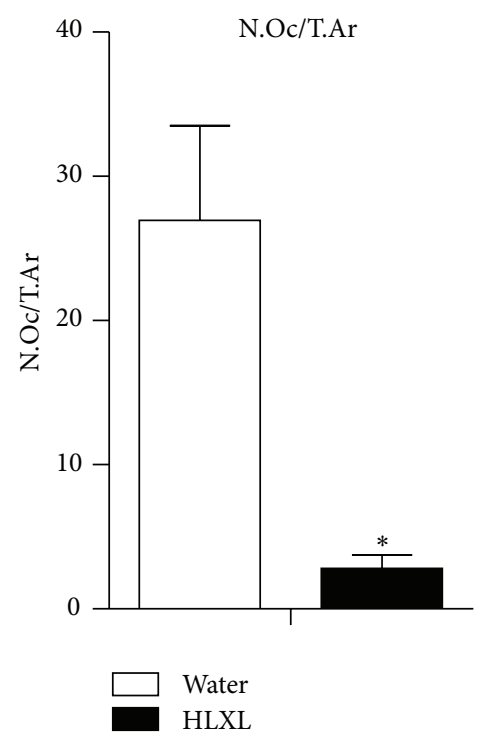

(b)

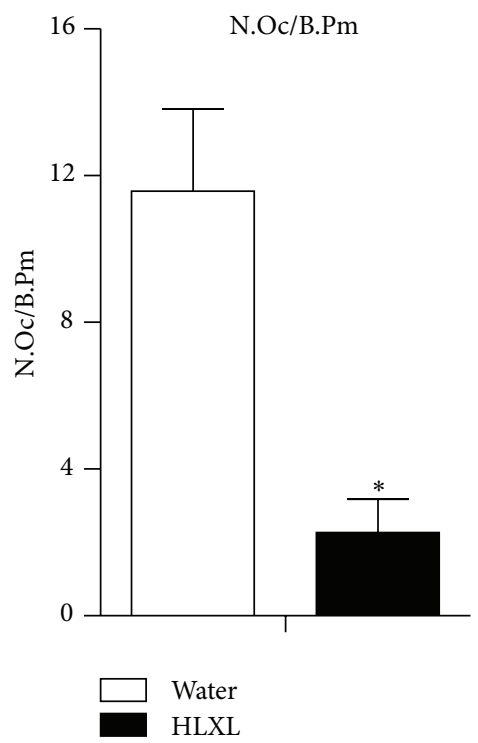

(d)

FIGURE 2: HLXL reduces bone resorption and osteoclast numbers in arthritic rats. Bone histomorphometry was performed on TRAP-stained sections of hind paws of HLXL-treated versus Water-treated rats $(n=5$ per group). The measurements of the following parameters are shown in the figure: (a) bone volume versus total tissue volume (BV/TV); (b) the number of osteoclasts per tissue area (N.Oc/T.Ar); (c) active resorption per bone surface area based on the ratio of osteoclast surface/bone surface area (Oc.S/BS); and (d) the number of osteoclasts per bone perimeter (N.Oc/B.Pm). $\left({ }^{*} P<0.05\right.$, comparing experimental and control samples.)

The levels of these MMPs were reduced in HLXL-treated rats compared to controls.

\section{Discussion}

Herbal TCM and other CAM modalities of the traditional systems of medicine have long been used for the treatment of RA and other inflammatory disorders in different parts of the world [31-34]. The use and popularity of CAM products have gradually been increasing in the western countries $[35,36]$. In this context, it is imperative that the composition of any herbal CAM to be considered for arthritis therapy is documented adequately. In addition, it is essential to define the mechanisms of action of herbal CAM to bring them to the mainstream of therapeutic arsenal for RA and other diseases. These priorities are essential to meet if herbal CAM is to be used as an adjunct to or in lieu of conventionally used drugs for RA. In a previous study, we have described the HPLC profile of HLXL and identified various compounds isolated, including steroids, terpenes, alkaloids, flavonoids, glycosides, and acids [23]. In another set of studies, we have elaborated the anti-inflammatory properties of HLXL $[11,13]$. In this 

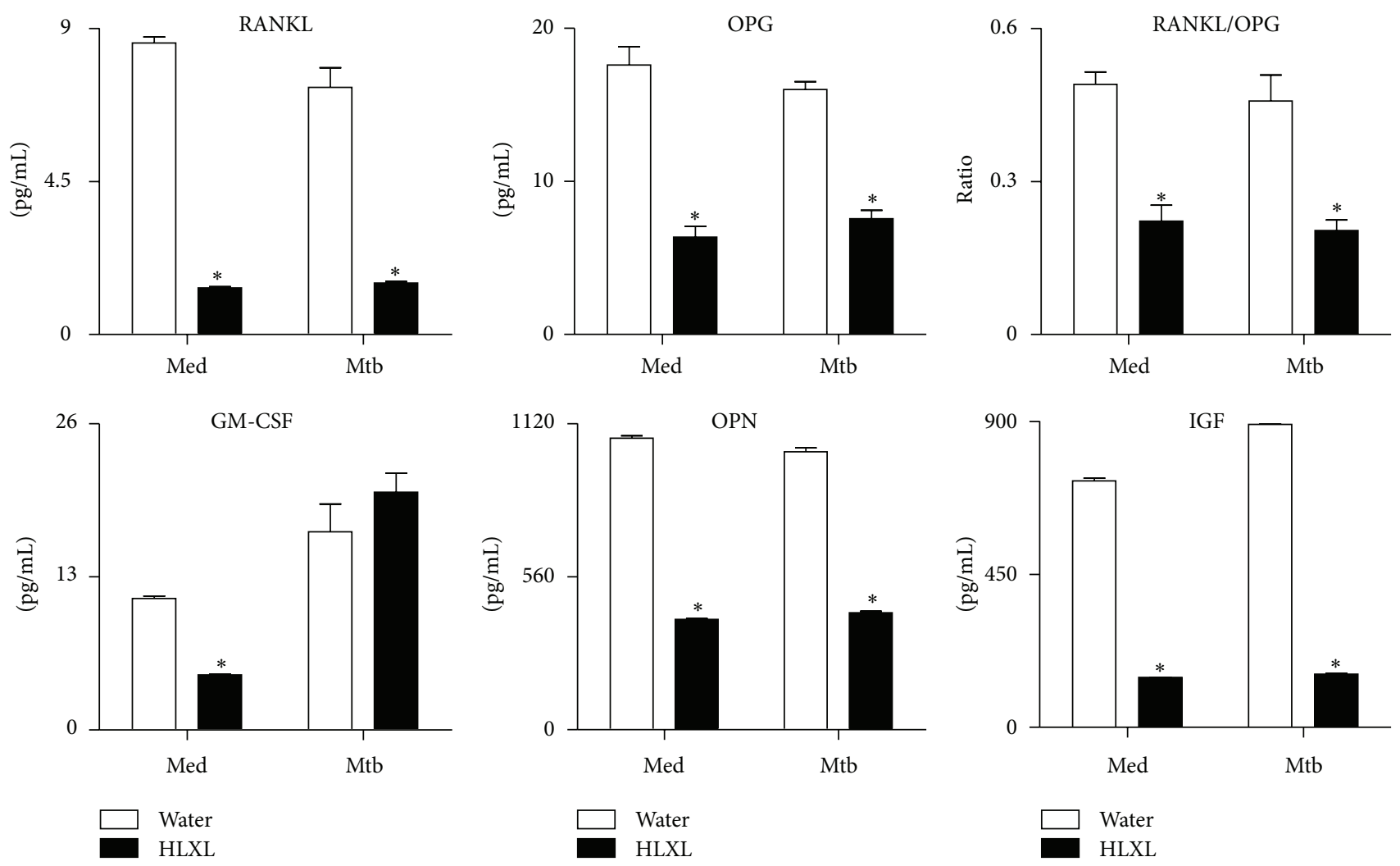

FIGURE 3: HLXL regulates mediators of bone remodeling in the joints of arthritic rats. Synovial-infiltrating cells (SIC) were harvested on d 18 from Mtb-immunized rats ( $n=4$ per group) treated with HLXL or Water (Vehicle) as described in Figure 1, and then these cells were restimulated for $24 \mathrm{~h}$ with $\mathrm{Mtb}$ sonicate $(10 \mu \mathrm{g} / \mathrm{mL})$. The levels of the indicated mediators were measured in culture supernatants of SIC using a Multiplex assay and the results were expressed as $\mathrm{pg} / \mathrm{mL} .{ }^{*} \mathrm{P}<0.05$, comparing experimental and control samples. (RANKL: receptor activator of nuclear factor kappa-B ligand; OPG: osteoprotegerin; GM-CSF: granulocyte-macrophage colony-stimulating factor; IGF: insulinlike growth factor; OPN: osteopontin; Med: medium; Mtb: heat-killed M. tuberculosis H37Ra.)

study, we have taken the first steps to fulfill above obligations regarding the use of HLXL, a Chinese herbal mixture, for the treatment of experimental arthritis in Lewis rats.

Our results show that treatment of arthritic Lewis rats with HLXL significantly reduced inflammation of hind paws as assessed by arthritic scores. This effect was further confirmed by histopathological examination of hind paw joints. Importantly, HLXL treatment also afforded protection against bone and cartilage damage. This was validated by histopathological and radiological examination of hind paws and finally confirmed by histomorphometry, which showed that HLXL-treated rats had higher bone mass, reduced bone resorption, and increased number of osteoclasts in the tissue section observed compared to control (Water-treated) rats. Thus, HLXL was effective in reducing both inflammation and bone damage in arthritic joints.

Bone remodeling is a balance of bone-forming (osteoblastic) and bone-resorping (osteoclastic) activities [37, 38]. Osteoclast-regulated bone remodeling is critically dependent on the activity of RANKL-RANK-OPG axis. RANKL is a tumor necrosis factor ligand superfamily member, and it is produced by osteoblasts [39]. RANKL binds to its cognate receptor RANK, which is expressed on osteoclast progenitors, mature osteoclasts, and chondrocytes [40-42]. The expression of RANKL can be induced by proinflammatory cytokines such as TNF- $\alpha$, IL-1 $\beta$, IL-6, and IL-17. RANKL acting with M-CSF is critical for the process of osteoclastogenesis and it influences the activation, maturation, and survival of osteoclasts. M-CSF is mainly produced by mature osteoblasts and it binds to colony-stimulating factor 1 receptor (c-fms) expressed on the surface of osteoclast precursors [43]. Other cellular sources of M-CSF are chondrocytes and synovial fibroblasts. IL- 1 and TNF- $\alpha$ promote M-CSF production. OPG, also known as osteoclastogenesis inhibitory factor (OCIF), is a soluble protein that serves as a decoy receptor for RANKL $[44,45]$. OPG competes with RANK for binding to RANKL, and it inhibits the maturation and activation of osteoclasts [39]. Soluble RANKL may not be a good indicator of bone loss as most RANKL is membrane bound. Interestingly, our results showed that HLXL-treated rats had a deviation of the RANKL/OPG ratio in favor of antiosteoclastic activity compared to Water-treated rats.

Additional mediators of bone remodeling include GMCSF, OPN, and IGF [46-48]. GM-CSF is produced by a variety of cells including macrophages, $\mathrm{T}$ cells, endothelial cells, and fibroblasts. It regulates the fusion of mononuclear osteoclasts into bone-resorbing osteoclasts [49]. OPN is produced by synovial fibroblasts, and it facilitates osteoclastic activity 

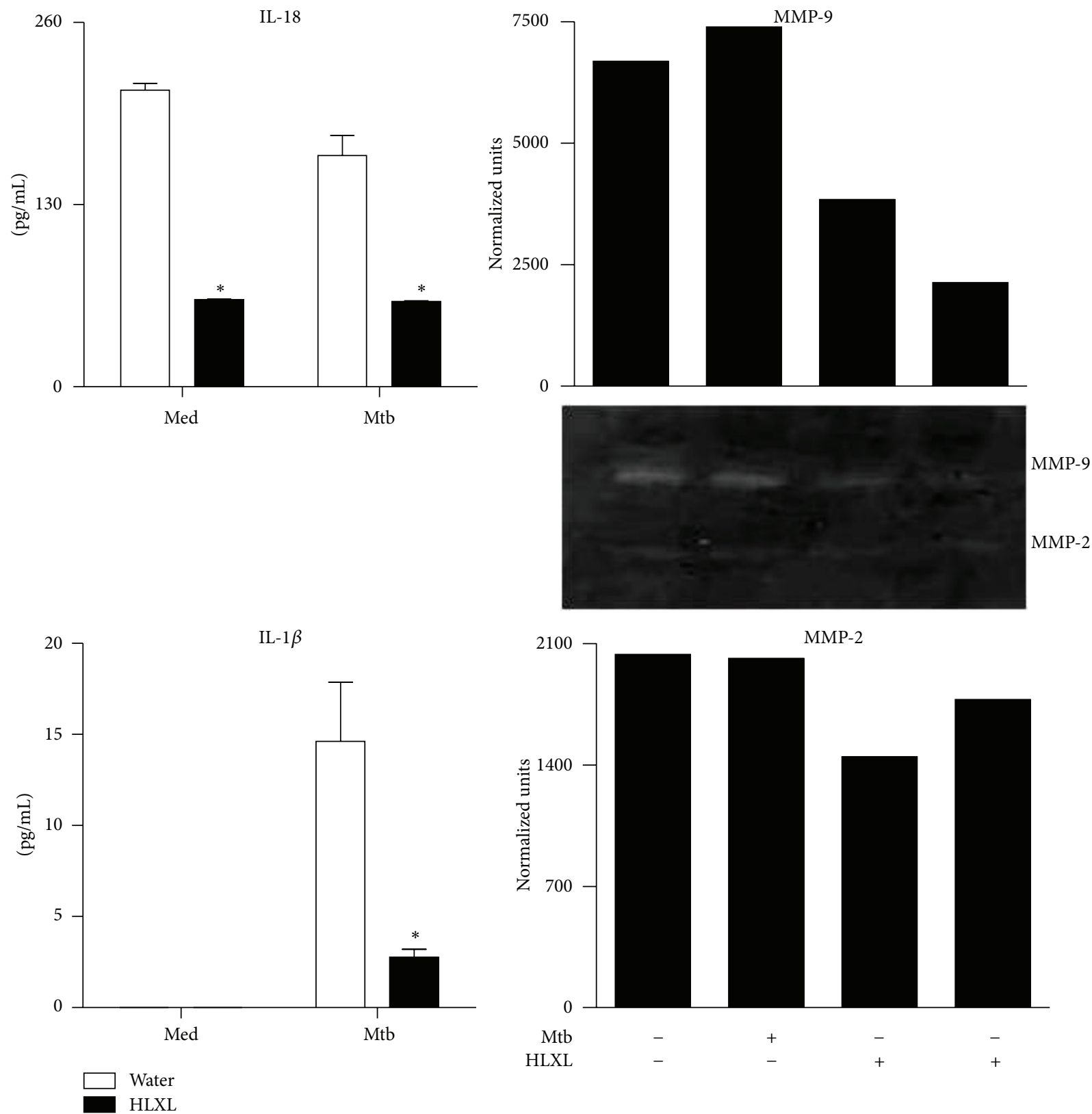

FIGURE 4: Inhibition of proinflammatory cytokines and MMP activity in HLXL-treated arthritic rats. Synovial-infiltrating cells (SIC) harvested on d 18 (peak phase of arthritis) from HLXL-fed or Water-fed rats ( $n=4$ per group) were cultured for $24 \mathrm{~h}$ with or without sonicated Mtb. The levels of cytokines IL-18 and IL-1 $\beta$ (left panel) in culture supernatants were measured using a Multiplex assay and the results were expressed as pg/mL. MMP-9 and MMP-2 activity (right panel) was tested in the culture supernatants of Mtb-restimulated SIC cells of HLXL/Water-treated rats. Mtb: heat-killed M. tuberculosis H37Ra; Med: medium. $\left({ }^{*} P<0.05\right.$, comparing experimental and control samples.)

while suppressing osteoblastic activity [48]. In addition, OPN can enhance angiogenesis [50] as well as production of proinflammatory cytokines IL- 6 and IL-17 [51, 52]. IGF mediates bone and cartilage degradation. Increased amounts of IGF are present in the synovial fluid of RA patients [47]. Interestingly, HLXL treatment significantly reduced the levels of GM-CSF, OPN, and IGF. Taken together with the results of altered RANKL/OPG ratio, these results show that HLXL treatment modulated the levels of the key mediators of bone remodeling (Figure 5).
The proinflammatory cytokines are among the vital inducers of some of the mediators of bone remodeling. Prominent among these cytokines are TNF- $\alpha$, IL- $1 \beta$, IL- 6 , IL17 , and IL-18. In this study, we showed that HLXL treatment significantly reduced the production of IL-1 $\beta$ and IL-18 by synovial-infiltrating cells compared to Vehicle (Water) treatment. In our previous study using HLXL, we showed that HLXL reduced the production of IL-6 and IL-17 [11]. However, in that study, we had measured the effect of HLXL on inflammation component of arthritis but not on the bone 


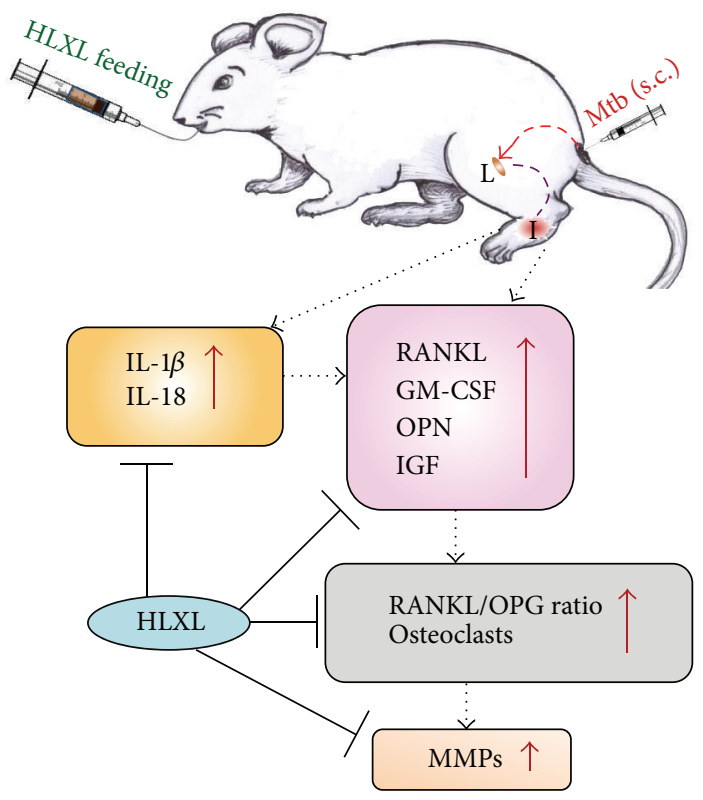

FIGURE 5: A schematic representation of the mechanisms involved in HLXL-induced suppression of bone damage in AA. HLXL suppressed bone damage via inhibiting the expression of osteoclastogenic mediators (receptor activator of nuclear factor kappa-B ligand: RANKL; granulocyte-macrophage colony-stimulating factor: GM-CSF; osteopontin: OPN; and insulin-like growth factor: IGF), cytokines (IL-18 and IL-1 $\beta$ ), and matrix metalloproteinases (MMPs). (L: lymph node, I: inflamed joint, Mtb: heat-killed Mycobacterium tuberculosis H37Ra, osteoprotegerin: OPG.) Red arrows highlight increased amount/activity of the indicated mediators in arthritis, which are inhibited by HLXL treatment.

remodeling parameters. The present study has filled that gap. Further, our results on MMP testing in HLXL-treated rats in SIC are supported by our earlier finding of HLXLinduced reduction in MMPs in spleen adherent cells (SAC) of arthritic rats [11]. Taken together, the results of our present and previous studies show that HLXL has a prominent effect on bone remodeling in part via reducing the production of various proinflammatory cytokines and MMPs (Figure 5).

\section{Conclusion}

In summary, our results demonstrate the antiarthritic activity of a Chinese herbal mixture, HLXL. Realizing the significance of properly documenting the composition of an herbal CAM, we performed our study using a well-characterized herbal mixture, HLXL. Further, to enhance the confidence of the public as well as the professionals in the rational use of a herbal mixture, we have invested effort in examining the mechanism by which HLXL protects against bone damage in arthritic joints (Figure 5). On the basis of the results of our study reported here combined with those of our previous studies on HLXL, we conclude that HLXL targets both inflammation and bone damage components of autoimmune arthritis, and that HLXL should be further evaluated in a preclinical study in RA patients.

\section{Conflict of Interests}

The authors declare that they do not have any financial conflict of interests.

\section{Acknowledgments}

This study was supported by Grants (P01 AT00260505, P.I.: Berman and R01 AT004321, P.I.: Moudgil) from the National Center for Complementary and Alternative Medicine (NCCAM) at the National Institutes of Health, Bethesda, MD, USA. The authors thank Professor Chen Shi-lin, (IMPLAD, Chinese Academy of Medical Sciences, Beijing, China) for his assistance in the acquisition and authentication of herbal source materials; Dr. Joseph Stains for his help and guidance in bone histomorphometry; Lisa Hester for her help in Multiplex assay; and Shivaprasad $\mathrm{H}$. Venkatesha, Brian Astry, Hua Yu, Lixing Lao, and Ruixin Zhang (University of Maryland) for their helpful discussions.

\section{References}

[1] P. E. Lipsky, "Rheumatoid arthritis," in Harrison's Principles of Internal Medicine, A. Fauci, E. Braunwald, D. Kasper et al., Eds., vol. Part 14, chapter 314, pp. 2083-2092, McGraw Hill, New York, NY, USA, 17th edition, 2008.

[2] C. L. Gorman and A. P. Cope, "Immune-mediated pathways in chronic inflammatory arthritis," Best Practice Research Clinical Rheumatology, vol. 22, no. 2, pp. 221-238, 2008.

[3] H. M. Kremers, P. Nicola, C. S. Crowson, W. M. O'Fallon, and S. E. Gabriel, "Therapeutic strategies in rheumatoid arthritis over a 40-year period," Journal of Rheumatology, vol. 31, no. 12, pp. 2366-2373, 2004.

[4] G. A. FitzGerald, "Coxibs and cardiovascular disease," The New England Journal of Medicine, vol. 351, no. 17, pp. 1709-1711, 2004.

[5] D. Bensky, A. Ellis, and R. Barolet, Chinese Herbal Medicine: Formulas and Strategies, Eastland Press, Seattle, Wash, USA, 
2nd edition, 2009.

[6] D. M. Taibi and C. Bourguignon, "The role of complementary and alternative therapies in managing rheumatoid arthritis," Family \& Community Health, vol. 26, no. 1, pp. 41-52, 2003.

[7] S. H. Venkatesha, B. M. Berman, and K. D. Moudgil, "Herbal medicinal products target defined biochemical and molecular mediators of inflammatory autoimmune arthritis," Bioorganic and Medicinal Chemistry, vol. 19, no. 1, pp. 21-29, 2011.

[8] P. M. Barnes, B. Bloom, and R. L. Nahin, "Complementary and alternative medicine use among adults and children: United States, 2007," National Health Statistics Reports, no. 12, pp. 1-23, 2008.

[9] B. G. Wan and B. H. Tao, "An 87 cases' clinical observation, arthritis treated by modified Huo-Luo-Xiao-Ling Dan," Jiangxi Journal of Traditional Chinese Medicine, vol. 33, article 12, 2002.

[10] Y. C. Wang, S. Y. Ji, and R. Wang, "The clinical application of Huo-Luo-Xiao-Ling Dan," Jiangxi Journal of Traditional Chinese Medicine, vol. 30, article 53, 2002.

[11] S. M. Nanjundaiah, D. Y. Lee, Z. Ma et al., "Modified huoluo-xiao-ling dan suppresses adjuvant arthritis by inhibiting chemokines and matrix-degrading enzymes," Evidence-Based Complementary and Alternative Medicine, vol. 2012, Article ID 589256, 2012.

[12] R. Rajaiah, D. Y. W. Lee, Z. Ma et al., "Huo-Luo-Xiao-Ling Dan modulates antigen-directed immune response in adjuvantinduced inflammation," Journal of Ethnopharmacology, vol. 123, no. 1, pp. 40-44, 2009.

[13] Y. H. Yang, R. Rajaiah, D. Y. W. Lee et al., "Suppression of ongoing experimental arthritis by a Chinese herbal formula (HuoLuo-Xiao-Ling Dan) involves changes in antigen-induced immunological and biochemical mediators of inflammation," Evidence-based Complementary and Alternative Medicine, vol. 2011, Article ID 642027, 10 pages, 2011.

[14] J. E. Fonseca, H. Canhão, N. J. Tavares, M. Cruz, J. Branco, and M. V. Queiroz, "Persistent low grade synovitis without erosive progression in magnetic resonance imaging of rheumatoid arthritis patients treated with infliximab over 1 year," Clinical Rheumatology, vol. 28, no. 10, pp. 1213-1216, 2009.

[15] L. A. B. Joosten, M. M. A. Helsen, T. Saxne, F. A. J. Van De Loo, D. Heinegård, and W. B. Van Den Berg, "IL-1 $\alpha \beta$ blockade prevents cartilage and bone destruction in murine type II collagen-induced arthritis, whereas TNF- $\alpha$ blockade only ameliorates joint inflammation," Journal of Immunology, vol. 163, no. 9, pp. 5049-5055, 1999.

[16] E. M. Gravallese, "Bone destruction in arthritis," Annals of the Rheumatic Diseases, vol. 61, supplement2, pp. ii84-ii86, 2002.

[17] P. E. Lipsky, D. M. van der Heijde, S. Clair et al., "Infliximab and methotrexate in the treatment of rheumatoid arthritis," The New England Journal of Medicine, vol. 343, no. 22, pp. 1594-1602, 2000.

[18] E. Lubberts, L. A. B. Joosten, M. Chabaud et al., "IL-4 gene therapy for collagen arthritis suppresses synovial IL-17 and osteoprotegerin ligand and prevents bone erosion," Journal of Clinical Investigation, vol. 105, no. 12, pp. 1697-1710, 2000.

[19] J. S. Smolen, C. Han, M. Bala et al., "Evidence of radiographic benefit of treatment with infliximab plus methotrexate in rheumatoid arthritis patients who had no clinical improvement: a detailed subanalysis of data from the anti-tumor necrosis factor trial in rheumatoid arthritis with concomitant therapy study," Arthritis and Rheumatism, vol. 52, no. 4, pp. 1020-1030, 2005.
[20] A. Y. Fan, L. Lao, R. X. Zhang, A. N. Zhou, and B. M. Berman, "Preclinical safety evaluation of the aqueous acetone extract of Chinese herbal formula Modified Huo Luo Xiao Ling Dan," Zhong Xi Yi Jie He Xue Bao, vol. 8, no. 5, pp. 438-447, 2010.

[21] L. Lao, A. Y. Fan, R. X. Zhang et al., "Anti-hyperalgesic and antiinflammatory effects of the modified Chinese herbal formula Huo Luo Xiao Ling Dan (HLXL) in rats," The American Journal of Chinese Medicine, vol. 34, no. 5, pp. 833-844, 2006.

[22] R. X. Zhang, A. Y. Fan, A. N. Zhou et al., "Extract of the Chinese herbal formula Huo Luo Xiao Ling Dan inhibited adjuvant arthritis in rats," Journal of Ethnopharmacology, vol. 121, no. 3, pp. 366-371, 2009.

[23] H. Cao, R. Yu, Y. Choi et al., "Discovery of cyclooxygenase inhibitors from medicinal plants used to treat inflammation," Pharmacological Research, vol. 61, no. 6, pp. 519-524, 2010.

[24] S. A. Komeh-Nkrumah, S. M. Nanjundaiah, R. Rajaiah, H. Yu, and K. D. Moudgil, "Topical dermal application of essential oils attenuates the severity of adjuvant arthritis in Lewis rats," Phytotherapy Research, vol. 26, no. 1, pp. 54-59, 2011.

[25] R. Rajaiah, M. Puttabyatappa, S. K. Polumuri, and K. D. Moudgil, "Interleukin-27 and interferon- $\gamma$ are involved in regulation of autoimmune arthritis," Journal of Biological Chemistry, vol. 286, no. 4, pp. 2817-2825, 2011.

[26] H. Yu, Y. H. Yang, R. Rajaiah, and K. D. Moudgil, "Nicotineinduced differential modulation of autoimmune arthritis in the lewis rat involves changes in interleukin-17 and anti-cyclic citrullinated peptide antibodies," Arthritis and Rheumatism, vol. 63, no. 4, pp. 981-991, 2011.

[27] S. M. Nanjundaiah, S. H. Venkatesha, H. Yu, L. Tong, J. P. Stains, and K. D. Moudgil, "Celastrus and its bioactive celastrol protect against bone damage in autoimmune arthritis by modulating osteoimmune cross-talk," Journal of Biological Chemistry, vol. 287, no. 26, pp. 22216-22226, 2012.

[28] R. S. Sellers, D. Peluso, and E. A. Morris, “The effect of recombinant human bone morphogenetic protein-2 (rhBMP-2) on the healing of full-thickness defects of articular cartilage," Journal of Bone and Joint Surgery A, vol. 79, no. 10, pp. 1452-1463, 1997.

[29] D. J. Chung, C. H. M. Castro, M. Watkins et al., "Low peak bone mass and attenuated anabolic response to parathyroid hormone in mice with an osteoblast-specific deletion of connexin43," Journal of Cell Science, vol. 119, no. 20, pp. 4187-4198, 2006.

[30] A. M. Parfitt, M. K. Drezner, F. H. Glorieux et al., "Bone histomorphometry: standardization of nomenclature, symbols, and units. Report of the ASBMR Histomorphometry Nomenclature Committee," Journal of Bone and Mineral Research, vol. 2, no. 6, pp. 595-610, 1987.

[31] P. M. Barnes, E. Powell-Griner, K. McFann, and R. L. Nahin, "Complementary and alternative medicine use among adults: United States, 2002," Advance Data, no. 343, pp. 1-19, 2004.

[32] J. Cibere, Z. Deng, Y. Lin et al., "A randomized double blind, placebo controlled trial of topical Tripterygium wilfordii in rheumatoid arthritis: reanalysis using logistic regression analysis," Journal of Rheumatology, vol. 30, no. 3, pp. 465-467, 2003.

[33] Y. Hijikata, Y. Miyamae, H. Takatsu, and S. Sentoh, "Two Kampo medicines, Jidabokuippo and Hachimijiogan alleviate sprains, bruises and arthritis," Evidence-based Complementary and Alternative Medicine, vol. 4, no. 4, pp. 463-467, 2007.

[34] D. Khanna, G. Sethi, K. S. Ahn et al., "Natural products as a gold mine for arthritis treatment," Current Opinion in Pharmacology, vol. 7, no. 3, pp. 344-351, 2007.

[35] D. M. Eisenberg, R. B. Davis, S. L. Ettner et al., "Trends in alternative medicine use in the United States, 1990-1997: results 
of a follow-up national survey," Journal of the American Medical Association, vol. 280, no. 18, pp. 1569-1575, 1998.

[36] G. M. Kuo, S. T. Hawley, L. T. Weiss, R. Balkrishnan, and R. J. Volk, "Factors associated with herbal use among urban multiethnic primary care patients: a cross-sectional survey," BMC Complementary and Alternative Medicine, vol. 4, article 18, 2004.

[37] B. F. Boyce and L. Xing, "The RANKL/RANK/OPG pathway," Current Osteoporosis Reports, vol. 5, no. 3, pp. 98-104, 2007.

[38] B. F. Boyce and L. Xing, "Functions of RANKL/RANK/OPG in bone modeling and remodeling," Archives of Biochemistry and Biophysics, vol. 473, no. 2, pp. 139-146, 2008.

[39] D. H. Jones, Y. Y. Kong, and J. M. Penninger, "Role of RANKL and RANK in bone loss and arthritis," Annals of the Rheumatic Diseases, vol. 61, supplement 2, pp. ii32-ii39, 2002.

[40] W. C. Dougall, M. Glaccum, K. Charrier et al., "RANK is essential for osteoclast and lymph node development," Genes and Development, vol. 13, no. 18, pp. 2412-2424, 1999.

[41] J. E. Fata, Y. Y. Kong, J. Li et al., “The osteoclast differentiation factor osteoprotegerin-ligand is essential for mammary gland development," Cell, vol. 103, no. 1, pp. 41-50, 2000.

[42] H. Hsu, D. L. Lacey, C. R. Dunstan et al., “Tumor necrosis factor receptor family member RANK mediates osteoclast differentiation and activation induced by osteoprotegerin ligand," Proceedings of the National Academy of Sciences of the United States of America, vol. 96, no. 7, pp. 3540-3545, 1999.

[43] F. P. Ross, "M-CSF, c-Fms, and signaling in osteoclasts and their precursors," Annals of the New York Academy of Sciences, vol. 1068, no. 1, pp. 110-116, 2006.

[44] W. S. Simonet, D. L. Lacey, C. R. Dunstan et al., "Osteoprotegerin: a novel secreted protein involved in the regulation of bone density," Cell, vol. 89, no. 2, pp. 309-319, 1997.

[45] H. Yasuda, N. Shima, N. Nakagawa et al., "Identity of osteoclastogenesis inhibitory factor (OCIF) and osteoprotegerin (OPG): A mechanism by which OPG/OCIF inhibits osteoclastogenesis in vitro," Endocrinology, vol. 139, no. 3, pp. 1329-1337, 1998.

[46] H. Franck, T. H. Ittel, O. Tasch, G. Herborn, and R. Rau, "Osteocalcin in patients with rheumatoid arthritis: a one-year followup study," Journal of Rheumatology, vol. 21, no. 7, pp. 12561259, 1994.

[47] P. A. Hill, J. J. Reynolds, and M. C. Meikle, "Osteoblasts mediate insulin-like growth factor-I and -II stimulation of osteoclast formation and function," Endocrinology, vol. 136, no. 1, pp. 124131, 1995.

[48] S. Ohshima, H. Kobayashi, N. Yamaguchi et al., "Expression of osteopontin at sites of bone erosion in a murine experimental arthritis model of collagen-induced arthritis: possible involvement of osteopontin in bone destruction in arthritis," Arthritis and Rheumatism, vol. 46, no. 4, pp. 1094-1101, 2002.

[49] M. S. Lee, H. S. Kim, J. T. Yeon et al., "GM-CSF regulates fusion of mononuclear osteoclasts into bone-resorbing osteoclasts by activating the Ras/ERK pathway," Journal of Immunology, vol. 183, no. 5, pp. 3390-3399, 2009.

[50] J. Dai, L. Peng, K. Fan et al., "Osteopontin induces angiogenesis through activation of PI3K/AKT and ERK1/2 in endothelial cells," Oncogene, vol. 28, no. 38, pp. 3412-3422, 2009.

[51] G. Chen, X. Zhang, R. Li et al., "Role of osteopontin in synovial Th17 differentiation in rheumatoid arthritis," Arthritis and Rheumatism, vol. 62, no. 10, pp. 2900-2908, 2010.

[52] Y. Take, K. Nakata, J. Hashimoto et al., "Specifically modified osteopontin in rheumatoid arthritis fibroblast-like synoviocytes supports interaction with B cells and enhances production of interleukin-6," Arthritis and Rheumatism, vol. 60, no. 12, pp. 3591-3601, 2009. 


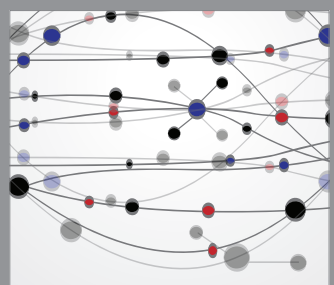

The Scientific World Journal
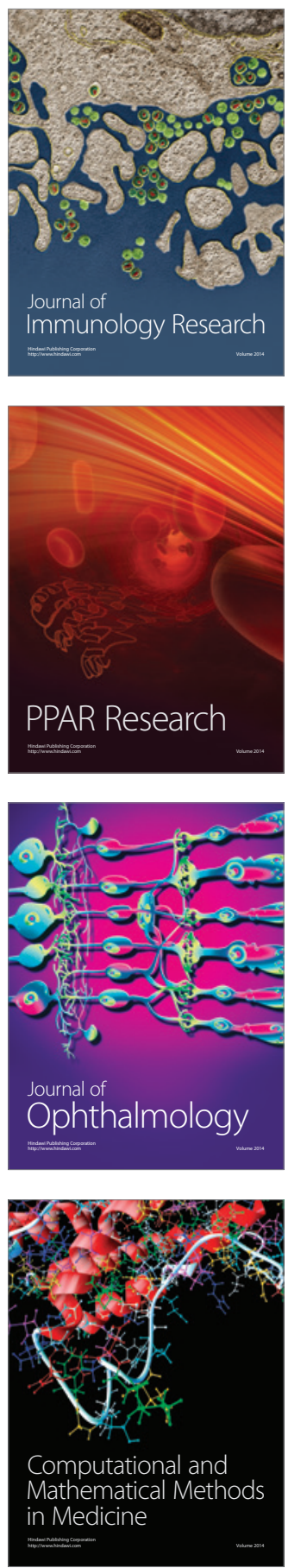

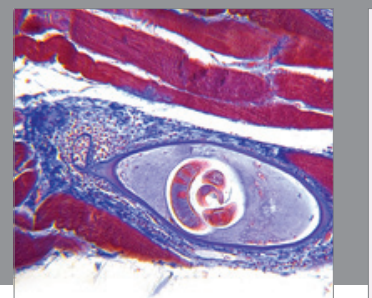

Gastroenterology

Research and Practice
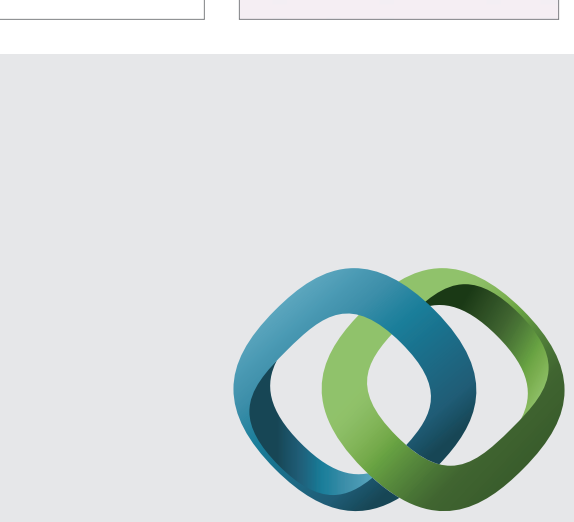

\section{Hindawi}

Submit your manuscripts at

http://www.hindawi.com
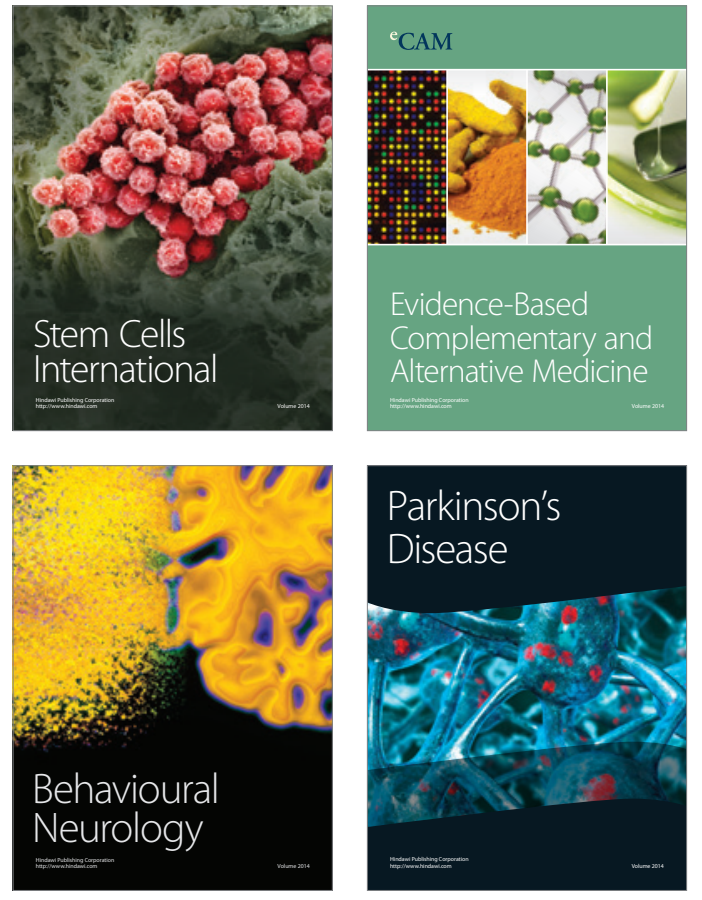
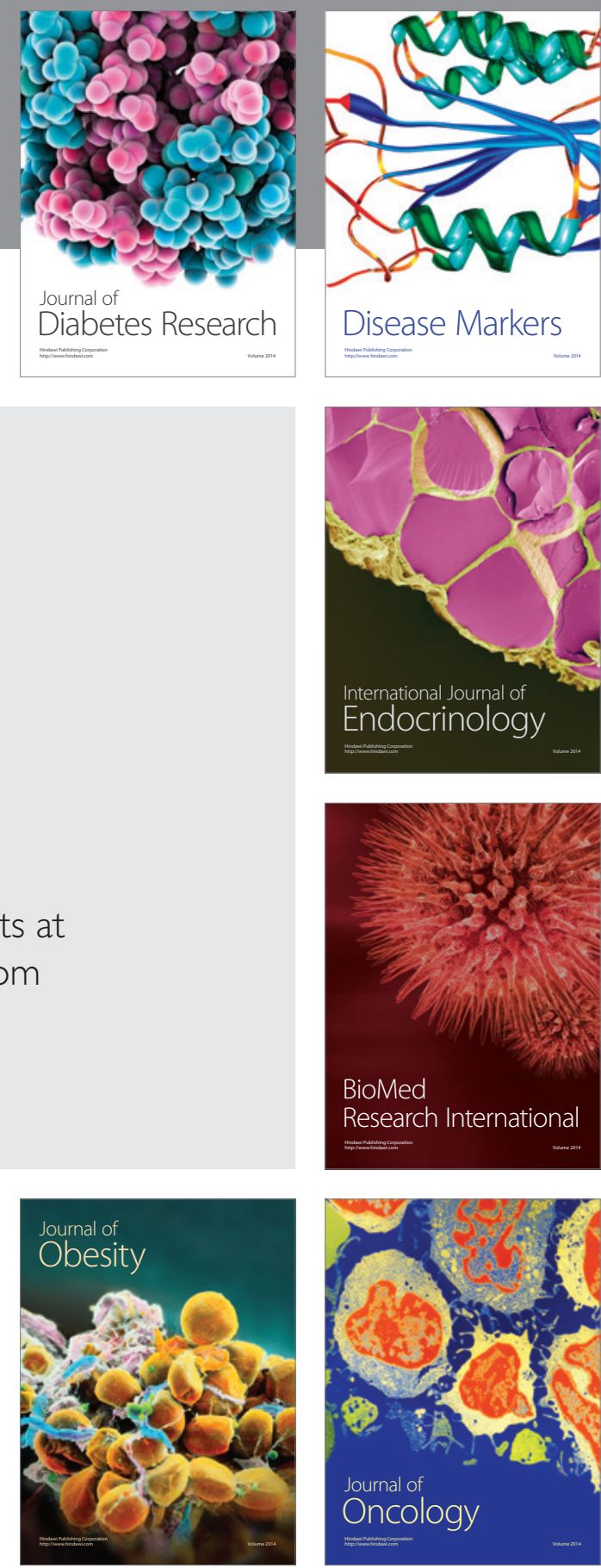

Disease Markers
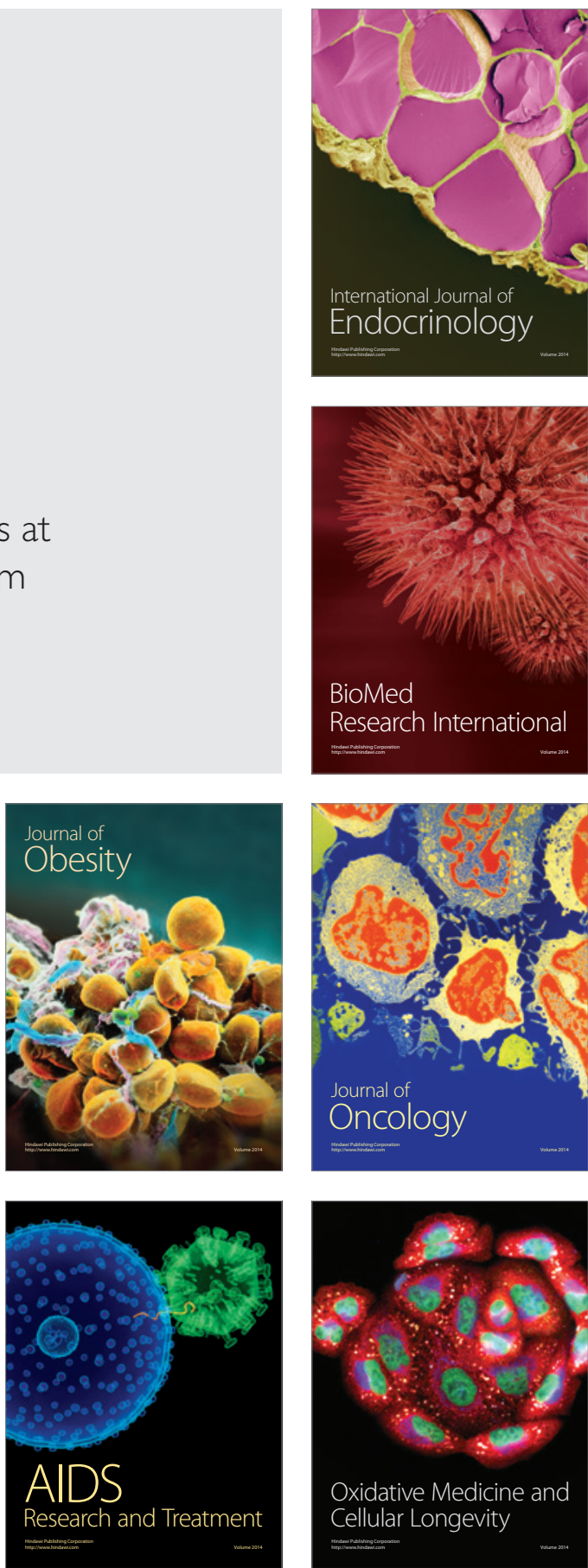